\title{
NURBS Skeleton: A New Shape Representation Scheme Using Skeletonization and NURBS Curves Modeling
}

\author{
Mohamed Naouai ${ }^{1,2}$, Atef Hammouda ${ }^{1}$, Sawssen Jalel ${ }^{1}$, and Christiane Weber ${ }^{2}$ \\ 1 Faculty of Science of Tunis, University campus el Manar DSI 2092 Tunis \\ Belvdaire-Tunisia Research unit Urpah \\ naouai@polytech.unice.fr, atef_hammouda@yahoo.fr, sawssen.jalel@gmail.com \\ 2 Laboratory Image and Ville UMR7011-CNRS-University Strasbourg 3rue de \\ l'Argonne F-67000 Strasbourg \\ naouai@polytech.unice.fr, christiane.weber@lorraine.u-strasbg.fr
}

\begin{abstract}
The representation and description of shapes or regions that have been segmented out of an image are early steps in the operation of most Computer vision systems; they serve as a precursor to several possible higher level tasks such as object/character recognition. In this context, skeletons have good properties for data reduction and representation. In this paper we present a novel shape representation scheme, named "NURBS Skeleton", based on the thinning medial axis method, the pruning process and the Non Uniform Rational B-Spline (NURBS) curves approximation for the modeling step.
\end{abstract}

Keywords: Skeleton, shape description, Medial Axis Transform (MAT), NURBS curves.

\section{Introduction}

Shape representation is one of the most important problems in pattern recognition and computer vision, and is an issue related to both data reduction and data description. Skeletons or Medial Axis Transform (MAT), as the shape descriptor, were described as having good properties for data reduction [1], [2].

There are two well-known paradigms for skeletonization methods: The first is that of "peeling an onion", i.e. iterative thinning of the original image until no pixel can be removed without altering the topological and morphological properties of the shape [5]. These methods require only a small number of lines in an image buffer at any time, which can be an advantage when dealing with large images. But on the other hand, multiple passes are necessary before reaching the final result, so that computation time may become quite high.

The second definition used for a skeleton is that of the ridge lines formed by the centers of all maximal disks included in the original shape, connected to preserve connectivity. This leads directly to the use of distance transforms or similar measures [13], [14], [15], which can be computed in only two passes on the image. 
The main drawback of traditional skeletons is their high sensitivity to noise in contour. Many methods have been proposed to stabilize the skeleton extraction, mostly by pruning "false" branches that are believed to be caused by noise in the outline [3], [4]. A different approach based on self-similarity of a smooth outline curve was presented in [9].

An efficient shape representation scheme using voronoi skeletons is described in [10]. This scheme possesses the important properties of connectivity as well as Euclidien metrics. Redundant skeletal edges are deleted in a pruning step which guarantees that connectivity of the skeleton will be preserved.

According to OGNIEWICZ and KUBLER [11], a robust and time-efficient skeletonization of a shape, which is connectivity preserving and based on Euclidian metrics, can be achieved by first regularizing the voronoi diagram (VD) of a shape's boundary points, i.e. by removal of noise sensitive parts of the tessellation and then by establishing a hierarchic organization of skeleton constituents. Each component of the VD is attributed with a measure of prominence which exhibits the expected invariance under geometric transformations and noise.

Couprie et al. [12], proposed a new definition and an algorithm for the discrete bisector function, which is an important tool for analyzing and filtering Euclidian skeletons. They introduced a new thinning algorithm which produces homotopic discrete skeletons. These algorithms, which are valid both in $2 \mathrm{D}$ and $3 \mathrm{D}$, are integrated in a skeletonization method which is based on exact transformations and allows the filtering of skeletons.

This paper is organized as follows. Section 2 presents NURBS curves. The proposed "NURBS Skeleton" is detailed in section 3. Working examples are illustrated in section 4. Finally we summarize our research in Section 5.

\section{NURBS Curves}

We recall that a NURBS curve of degree $\mathrm{p}$ is defined by:

$$
C(u)=\frac{\sum_{i=0}^{n} N_{i, p}(u) w_{i} P_{i}}{\sum_{i=0}^{n} N_{i, p}(u) w_{i}}
$$

Where $\left\{P_{i}\right\}$ are control points, $\left\{w_{i}\right\}$ are the weights associated with these points. Indeed, each $w_{i}$ determines the influence of point $P_{i}$ on the curve. And the $\left\{N_{i, p}(u)\right\}$ are the B-spline basis functions of degree $\mathrm{p}$ defined on the non-periodic and non-uniform knot vector $\mathrm{U}$, recursively by:

$$
N_{i, p}(u)=\frac{u-u_{i}}{u_{i+p}-u_{i}} N_{i, p-1}(u)+\frac{u_{i+p+1}-u}{u_{i+p+1}-u_{i+1}} N_{i+1, p-1}(u)
$$

Where

$$
N_{i, 0}(u)= \begin{cases}1 & \text { si } u_{i} \leq u<u_{i+1} \\ 0 & \text { sinon }\end{cases}
$$

The non-periodic knot vector is defined by $U=\left\{a, \ldots, a, u_{p+1}, \ldots, u_{m p-1}, b, \ldots, b\right\}$ with a multiplicity of $\mathrm{a}$ and $\mathrm{b}$ which is equal to the order of the curve. This 
constraint ensures the passage of the curve by the two endpoints. Throughout this paper, we assume that the parameter lies in the range $u \in[0,1]$ and the weight $w_{i}$ is set to 1 . For a detailed presentation of NURBS and its properties, we refer the reader to reference [6]. However, the main properties interesting for us in NURBS curves can be summarized into four points:

- Interpolation of extreme points.

- Affine invariance (translation, rotation, homothety)

- Convex hull: curve is still in the convex hull of its control points.

- Local modification: if a parameter (point, weight) is changed, curve is changed only within a certain interval.

\section{NURBS Skeleton}

\subsection{Skeleton Extraction}

The alternative approach we follow consists in creating a version of the object that is as thin as possible, i.e. thinning the object to a set of idealized thin lines. The resulting thin lines are called the skeleton or medial axis, of the input pattern and they are the thinnest representation of the original object that preserves the topology aiding synthesis and understanding. The methods to accomplish this, are called thinning or skeletonization.

In this first phase of our approach, skeleton of the considered shape is extracted using the algorithm described in [5]. Fig. 1 shows the result after applying skeleton's extraction algorithm.

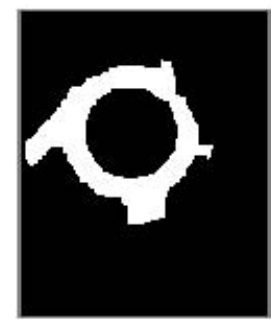

(a)

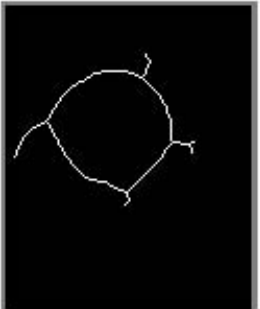

(b)

Fig. 1. (a) Initial shape. (b) Skeleton extraction.

\subsection{Interest Points Detection}

The detection of end points, junction points and curve points of medial axis is important for a structural description that captures the topological information embedded in the skeleton. The thin lines can be converted into a graph associating the curve points with the edges, the end and the junction points with the vertices. Such a skeletal graph can then be used as an input to graph matching 


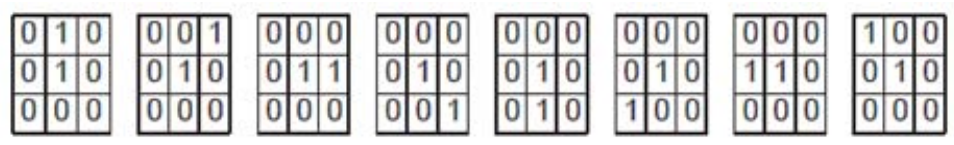

Fig. 2. SEs for the end points: the fundamental $A$ and its rotations $\left(\Theta_{1}(\mathrm{~A}), \Theta_{2}(\mathrm{~A}), \ldots, \Theta_{7}(\mathrm{~A})\right.$ in order

algorithms [7], [8]. In this paper, we introduce algorithms for detecting interest points or skeletons characteristic points (see Fig. 4) (end points, junction points and curve points) based on a morphological approach.Formally we define the end points, the junction points as follows:

Definition 1: A point of one-pixel width digital curve is an end point if it has a single pixel among its $3 \times 3$ neighborhood.

Definition 2: A point of one-pixel width digital curve is defined as a junction point if it has more than two curve pixels among its $3 \times 3$ neighborhood.

We propose another, purely morphological, method to detect end points and junction points from a skeleton as follows. To extract the end points, we perform erosion transform with the complement of each SE defining an end point $\bar{A}$, and its rotations $\Theta_{i}(\bar{A})$. On the complement of $X(\bar{X})$, we take the union of all the results and then we intersect the union with $X$.

$$
\operatorname{ENDPOINTS}(X)=\left[\bigcup_{i} \varepsilon_{\Theta_{i}(\bar{A})}(\bar{X})\right] \bigcap X
$$

where $\varepsilon_{\Theta_{i}(\bar{A})}(\bar{X})$ denotes the erosion of $\bar{X}$ by $\Theta_{i}(\bar{A})$.

According to the definition of junction points, only curve pixels are considered in the neighboring configuration. In the eight-connected square grid, we can have two fundamental configurations corresponding to a junction point, $B$ and $C$, and their seven rotations of $45^{\circ}$ (Fig. 3). Thus, the extraction of the junction points from a skeleton is obtained by performing erosion transforms with each $\operatorname{SE}(B, C)$ and their rotations $\Theta_{i}(B)$ and $\Theta_{i}(C)$. and then taking the union of the results:

$$
J U N C T I O N P O I N T S(X)=\left[\bigcup_{i} \varepsilon_{\Theta_{i}(B)}(X)\right] \cup\left[\bigcup_{i} \varepsilon_{\Theta_{i}(C)}(X)\right]
$$

All the curve points are trivially obtained by removing the end points and the junction points from the skeleton.

\subsection{Edges Extraction and Pruning Process}

Skeletal pixels are classified into three sets which are the junction set (JS), the end set (ES) and the curve set (CS). Evidently, if $\mathrm{S}$ is the universal set corresponding to the skeleton, then: 


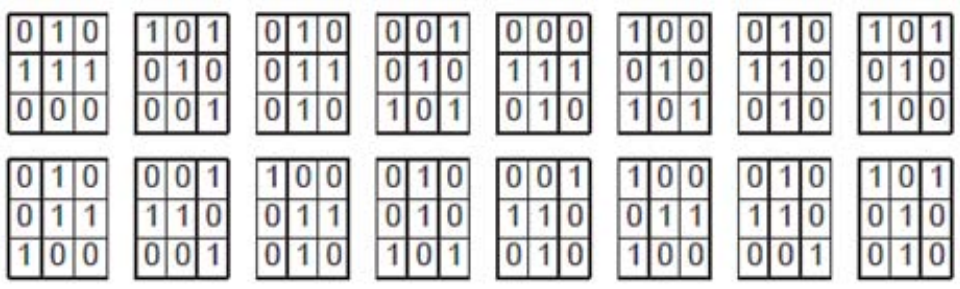

Fig. 3. SEs for the junction points: $\mathrm{B}$ and its rotations $\left(\Theta_{1}(\mathrm{~B}), \Theta_{2}(\mathrm{~B}), \ldots, \Theta_{7}(\mathrm{~B})\right.$ and $\mathrm{C}$ and its rotations $\left(\Theta_{1}(\mathrm{C}), \Theta_{2}(\mathrm{C}), \ldots, \Theta_{7}(\mathrm{C})\right.$

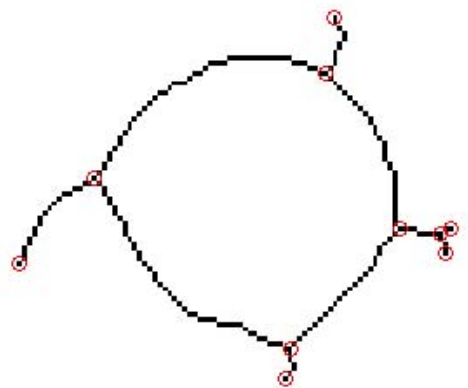

Fig. 4. Interest points detection

$$
S=J S \oplus E S \oplus C S
$$

Considering these sets, skeleton can be converted into a graph associating the curve points with the edges, the end and the junction points with the nodes. Thus, an edge is defined as the set of adjacent pixels which is limited between two nodes. Consequently, three types of edges can be considered, according to the type of their extreme nodes. They are:

External edges: both extreme nodes are end nodes.

Internal edges: both extreme nodes are junction nodes.

Branches: one node is an end node and the other one is a junction node.

Following the edge definition, an automatic search of skeletal edges is executed. Simultaneously, short skeletal branches which are not significant are removed. This latter is called the pruning process. Hence, skeleton is reduced and data is updated which requires to rerun the interest points detection process and the edges extraction process in order to save only significant edges.

\subsection{NURBS Curves Approximation}

In the previous step, the skeleton of the shape is partitioned in edges. The goal of this phase is to construct these objects by using NURBS curves approximation. We recall that to construct a NURBS curve of degree $p$, it is necessary to define 
$\mathrm{n}+1$ control points to approximate. Each point is identified by its coordinates in the plane and by its weight.

Therefore, the selection of control points and the scheduling of these points are inevitable to construct a NURBS curve. Our algorithm operates edge by edge, according to the following instructions:

1. Selects an edge E.

2. Determines the controls points of E.

3. Sets the NURBS curve parameters (degree p, knot vector U, weights W).

4. Generates the corresponding NURBS curve.

Depending on the size of the considered edge, a threshold value has to be introduced in order to subsample the edge's pixels set. In fact, this value must be, obviously, strictly less than the edge's size.

More the threshold value decreases, more the number of subsampled values increases. In our work, we set this value to 5 for the edges of sizes greater than 15. Otherwise, it is fixed at 2.

In addition to these subsampled values, edge's extreme nodes are included in the set of control points. This ensures the continuity between different edges. Thus, for each edge, we define the set of controls points $\mathrm{P}$ as:

$$
P=\{\text { Extreme Nodes }\} \bigcup\{\text { SubsampledValues }\}
$$

\section{Experimental Results}

The experimental results of the NURBS Skeleton are shown in the following figures. In fact our method produces smooth curves representing the shape's skeleton where non significant information is deleted unlike the Thinning medial axis method [5] and the voronoi medial axis method [1].

In Fig. 7, the Fig. 7(a) presents the original binary image. Fig. 7(b) is the skeleton obtained by the Thinning medial axis method and Fig. 7(c) is the result of the NURBS Skeleton method. In this example five branches have been erased.

In Fig. 8, two branches have been erased. These latter corresponds to the goat's feet and are significant in this shape representation. Hence, by increasing the threshold value for the pruning process, we risk losing useful information. Nevertheless, the shape obtained by our method is more smooth with few inflection points.

Fig. 9 shows an example of an airplane with some small salient subparts. Fig. 9 (b) is the skeleton generated by the Thinning medial axis method and does not look like an airplane. Fig. 9(c) is the skeleton generated by our method that some small subparts were removed. The skeleton can be recognized easily as an airplane.

The experimental results are very promising both in shape representation and shape reduction. In fact, Table. 1 presents reduction factors of tested shapes. This factor is calculated as:

$$
R F=\frac{\text { Interest points number obtained by Thinning Medial Axis }}{\text { Interest points number obtained by NURBS Skeleton }}
$$




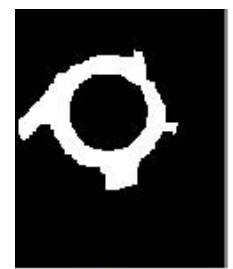

(a)

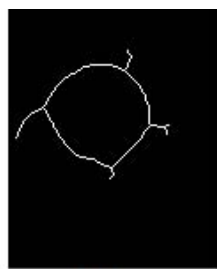

(b)

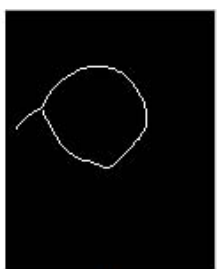

(c)

Fig. 5. Example of closed shape: (a) Original shape. (b) Thinning medial axis. (c) NURBS Skeleton.

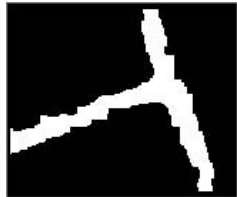

(a)

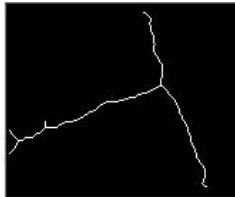

(b)

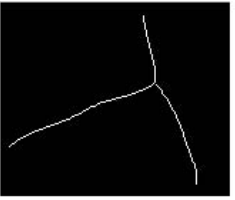

(c)

Fig. 6. Example of T shape: (a) Original shape. (b) Thinning medial axis. (c) NURBS Skeleton.

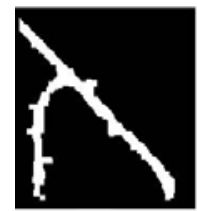

(a)

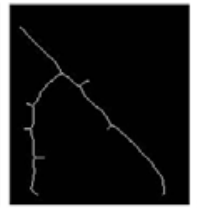

(b)

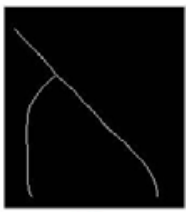

(c)

Fig. 7. Example of Y shape: (a) Original shape. (b) Thinning medial axis. (c) NURBS Skeleton.

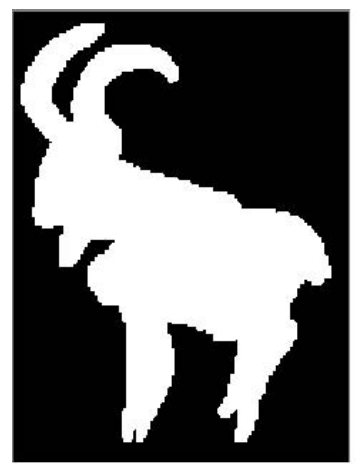

(a)

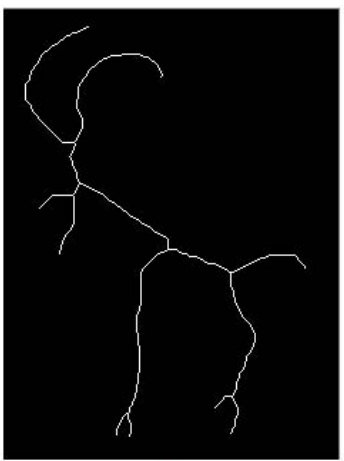

(b)

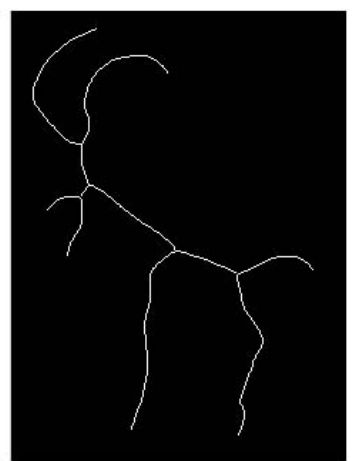

(c)

Fig. 8. Example of a goat: (a) Original shape. (b) Thinning medial axis. (c) NURBS Skeleton. 


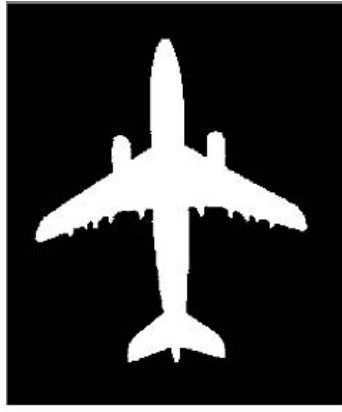

(a)

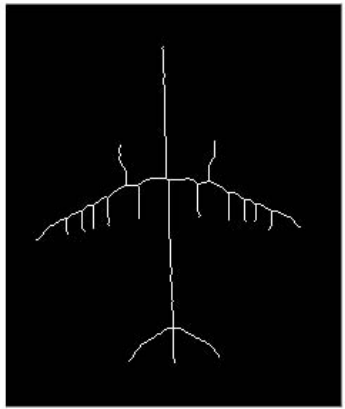

(b)

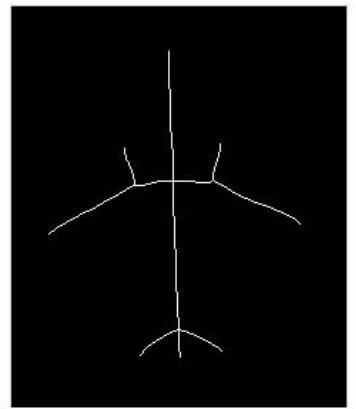

(c)

Fig. 9. Example of an airplane: (a) Original shape. (b) Thinning medial axis. (c) NURBS Skeleton.

Table 1. Interest points number and reduction factor

\begin{tabular}{|c|c|c|c|}
\hline Images & Thinning Medial Axis & NURBS Skeleton & Reduction factor (RF) \\
\hline Closed shape & 10 & 2 & 5 \\
\hline T shape & 8 & 4 & 2 \\
\hline Y shape & 14 & 4 & 3.5 \\
\hline Goat & 16 & 12 & 1.3 \\
\hline Airplane & 32 & 12 & 2.6 \\
\hline
\end{tabular}

\section{Conclusion}

We describe in this paper a novel skeleton extraction algorithm in binary images for shape representation. This algorithm named "NURBS Skeleton" is based on the thinning medial axis method, the pruning process and the Non Uniform Rational B-Spline (NURBS) curves approximation for the modeling step. This novel method has produced good results for data reduction by the use of pruning process.However, increasing the threshold value for the pruning process may cause losing useful information. Good results are also obtained for data representation by emphasizing the wealth of NURBS modeling in the field of pattern recognition.

\section{References}

1. Mang, C., Yun-cai, L.: Connection Skeleton Extraction Based on Contour Connectedness. Shanghai Jiaotong Univ. (Sci.) 13(5), 521-527 (2008)

2. Pavaldis, T.: A review of algorithms for shape analysis. Computer Graphics and Image Processing 7, 243-258 (1978)

3. Kimmel, R.: Skeletonization via distance maps and level sets. Computer Vision and Image Understanding 62(3), 382-391 (1995)

4. Brandt, J.W., Algazi, V.R.: Continuous skeleton computation by voronoi diagram. CVGIP: Image Understanding 55, 329-338 (1994) 
5. Lam, L., Lee, S.-W., Suen, C.Y.: Thinning Methodologies-A Comprehensive Survey. IEEE Transactions on Pattern Analysis and Machine Intelligence 14(9), 879 (1992)

6. Piegl, L., Tiller, W.: The NURBS Book. 2nd edn.

7. Messmer, B.T., Bunke, H.: EFcient subgraph isomorphism detection: a decomposition approach. IEEE Trans. Knowledge Data Eng. 12(2), 307-323 (2000)

8. Ruberto, D., Rodriguez, G., Casta, L.: Recognition of shapes bymorphological attributed relational graphs. In: Atti dellVIII Convegno AIIA 2002, Siena, Italy (2002); Messmer, B.T., Bunke, H.: EFcient subgraph isomorphism

9. Liu, T.L., Geiger, D., Kohn, R.V.: Representation and self similarity of shape. In: Proceedings of the Sixth International Conference on Computer Vision, pp. 1129-1135. IEEE Computer Society, Washington (1999)

10. Niranjan, M., Rajan, V.T.: An efficient shape representation scheme using Voronoi skeletons. Pattern Recognition Letters 16, 147-160 (1995)

11. Ogniewicz, R.L., Kbler, O.: Hierarchic Voronoi Skeletons. Pattern Recognition 28(3), 343-359 (1995)

12. Couprie, M., Coeurjolly, D., Zrour, R.: Discrete bisector function and Euclidian skeleton in 2D and 3D. Image and Vision Computing 25, 1543-1556 (2007)

13. Borgefors, G.: Distance Transforms in Digital Images. Computer Vision, Graphics and Image Processing 34, 344-371 (1986)

14. Chiang, J.Y., Tue, S.C., Leu, Y.C.: A New Algorithm for Line Image Vectorization. Pattern Recognition 31(10), 1541-1549 (1998)

15. Niblack, C.W., Gibbons, P.B., Capson, D.W.: Generating Skeletons and Centerlines from the Distance Transform. CVGIP: Graphical Models and Image Processing 54(5), 420-437 (1992) 